TRABAJOS ORIGINALES

Rev Obstet Ginecol Venez. 2021; 81 (4): 314-318.

https://doi.org/10.51288/00810404

\title{
Cáncer de cuello uterino. Incidencia registrada en el Hospital "Dr. Domingo Luciani” en el año 2020
}

\author{
(iD) María Nastasi, ${ }^{1}$ (D) Ernesto Lara, ${ }^{2}$ (D) María Mercedes Pérez, ${ }^{3}$ (D) Elianny Betancourt, ${ }^{2}$ \\ (iD) Lucía Aguilera. ${ }^{1}$
}

\begin{abstract}
RESUMEN
Objetivo: Estimar la incidencia de cáncer de cuello uterino (CCU) en el año 2020 en la consulta de ginecología oncológica del Servicio de Obstetricia y Ginecología del Hospital "Dr. Domingo Luciani”.

Método: Estudio retrospectivo, descriptivo, donde se revisaron las historias clínicas de las pacientes con diagnóstico de cáncer de cuello uterino que acudieron a primera consulta de ginecología oncológica, el periodo de enero a diciembre 2020.

Resultados: Sesenta pacientes cumplieron los criterios de inclusión, las variables estudiadas fueron edad, tipo histológico, estadio al momento del diagnóstico y tratamiento indicado. Resultados: 14,52\% fueron diagnosticadas con cáncer de cuello uterino; la media de edad al momento del diagnóstico fue de 43,15 años; el grupo histológico más común resulto ser escamoso, (96,67\%). Con relación al estadiaje, 28 casos (46,67\%) se diagnosticaron en estadio IIIB, 14 casos $(23,33 \%)$ en estadio IIB y solo 6,66 \% fueron diagnosticadas en estadios precoces. El tratamiento indicado fue radioterapia y quimioterapia.

Conclusión: Se evidencia en el presente estudio que el cáncer de cuello uterino representa un porcentaje importante de consultas ginecológica, que lamentablemente el diagnóstico se hace en estadios avanzados, afectando negativamente el pronóstico de estas pacientes y que la edad en que predomina es en plena vida productiva de las mujeres afectadas, constituyendo un verdadero problema de salud pública y un caos para las familias y la sociedad.
\end{abstract}

Palabras clave: Cáncer de cuello uterino, Incidencia, Carcinoma, Adenocarcinoma, Estadio.

SUMMARY

Objective: To estimate the incidence of cervical cancer (CCU) in the year 2020 in the gynecology oncology consultation of the Obstetrics and Gynecology Service of the Hospital "Dr. Domingo Luciani".

Method: Retrospective, descriptive study, where the clinical histories of the patients diagnosed with cervical cancer in who attended the first oncology gynecology consultation were reviewed, the period from January to December 2020.

Results: Sixty patients met the inclusion criteria, the variables studied were age, histological type, stage at the time of diagnosis and indicated treatment. Results: $14.52 \%$ were diagnosed with cervical cancer; the mean age at the time of diagnosis was 43.15 years; the most common histological group was squamous, (96.67\%). Regarding staging, 28 cases (46.67\%) were diagnosed in stage IIIB, 14 cases (23.33\%) in stage IIB, and only $6.66 \%$ were diagnosed in early stages. Treatment indicated radiotherapy and chemotherapy.

Conclusion: We show in the present study that cervical cancer represents a significant percentage of gynecological consultations, that unfortunately the diagnosis is made in advanced stages, negatively affecting the prognosis of these patients and that the age at which it predominates is in full productive life of the women. affected women, constituting a real public health problem and chaos for families and society.

Keywords: Cervical cancer, Incidence, Carcinoma, Adenocarcinoma, Stage.

\begin{abstract}
${ }^{1}$ Residente del Tercer año de Postgrado de Ginecología y Obstetricia, Universidad Central de Venezuela. ${ }^{2}$ Ginecólogo Oncólogo, Ginecólogo Obstetra. Adjunto del Servicio de Ginecología Hospital "Dr. Domingo Luciani”, Caracas, Venezuela. ${ }^{3}$ Ginecólogo Infanto - Juvenil, Ginecólogo Obstetra. Jefe del Servicio de Ginecología. Hospital "Dr. Domingo Luciani”, Caracas, Venezuela. Jefe de servicio de Ginecología. Correo de correspondencia: marianastasib@gmail.com
\end{abstract}

Forma de citar este artículo: Nastasi M, Lara E, Pérez MM, Betancourt E, Aguilera L. Cáncer de cuello uterino. Incidencia registrada en el Hospital "Dr. Domingo Luciani” en el año 2020. Rev Obstet Ginecol Venez. 2021; 81(4):314-318. https://doi.org/10.51288/00810404

\section{INTRODUCCIÓN}

A nivel mundial, el cáncer de cuello uterino continúa siendo uno de los cánceres más comunes, con 604127 nuevos casos y 341831 muertes para el año 2020, esta enfermedad se ubica como la cuarta causa de incidencia y mortalidad por cáncer en las mujeres (1). La mayoría 


\section{CÁNCER DE CUELLO UTERINO. INCIDENCIA REGISTRADA EN EL HOSPITAL \\ “DR. DOMINGO LUCIANI” EN EL AÑO 2020}

de los nuevos casos y defunciones (aproximadamente, $85 \%$ y $90 \%$, respectivamente) ocurren en regiones de bajos recursos o sectores socioeconómicamente más bajos de la sociedad (2), ocupando en países de bajos ingresos, la segunda causa de incidencia y mortalidad por patología maligna (3). En Venezuela, para 2020, según la Agencia Internacional de Investigación en Cáncer (IARC), el cáncer de cuello uterino fue diagnosticado en 3709 mujeres, y fallecieron 2129 a causa de esta enfermedad, con una tasa de incidencia y mortalidad de 22,2 y 12,5 por cada 100000 mujeres respectivamente. Representa la segunda causa de incidencia y mortalidad por cáncer en la población femenina (4). Los genotipos de alto riesgo del virus papiloma humano (VPH) causan casi todos los cánceres de cuello uterino y los programas de detección y vacunación del VPH son una estrategia efectiva en la prevención de enfermedades (5). A pesar ser una patología potencialmente prevenible, continúa siendo un problema de salud pública en el mundo, especialmente para los países de bajos ingresos, cuyos sistemas de salud son de acceso limitado y recursos deficientes (6). El carcinoma de células escamosas y el adenocarcinoma son los subtipos histológicos más comunes y representan aproximadamente el $70 \%$ y el $25 \%$ de todos los cánceres de cuello uterino, respectivamente $(7,8)$. Anteriormente, la estadificación del cáncer de cuello uterino de la Federación Internacional de Ginecología y Obstetricia (FIGO) se basaba principalmente en el examen clínico. A partir de 2018, el Comité de Ginecología Oncológica de la FIGO modificó la clasificación anterior, permitiendo que los hallazgos patológicos y de imágenes, cuando estén disponibles, se utilicen para asignar el estadio. El tratamiento dependerá del estadio de la enfermedad, realizándose principalmente mediante cirugía o radioterapia, con la quimioterapia como un valioso complemento (2). El presente trabajo tiene como finalidad describir la situación actual del cáncer de cuello uterino en el Hospital Dr. Domingo Luciani, evaluando aspectos epidemiológicos, diagnósticos y de tratamiento; entendiendo el rol crítico y necesario que juega contar con datos que permitan informar sobre la evolución de esta patología en Venezuela.

\section{MÉTODOS}

Se trata de un estudio retrospectivo y descriptivo, en el cual se revisaron las historias clínicas de todas las pacientes con diagnóstico de cáncer de cuello uterino (CCU), que acudieron a la consulta de ginecología oncológica del Hospital "Dr. Domingo Luciani” entre los meses de enero a diciembre de 2020, fijándose como criterios de inclusión el diagnóstico histológico de cáncer de cuello uterino. La muestra quedó constituida por 60 historias clínicas, recolectándose los datos referentes a la edad, tipo histológico, estadio FIGO y tratamiento indicado. Los datos fueron tratados con técnicas propias de estadística descriptiva y los resultados aquí presentados se circunscriben a la muestra. Las variables se describieron en términos de frecuencias absolutas y relativas y se expresaron en forma de gráficos.

\section{RESULTADOS}

Para 2020, en la consulta de ginecología oncológica del hospital Dr. Domingo Luciani, la incidencia de cáncer de cuello uterino correspondió a 60 pacientes de 413 que acudieron por primera vez, representado un $14,52 \%$ del total de todas las consultas de primera (Gráfico 1).

El grupo etario más afectado fue el comprendido entre los 41 a 50 años con $31,6 \%$ de los casos, seguidos por el grupo entre 31 a 40 años con $30,0 \%$. Las pacientes menores de 50 años se vieron afectadas en más del $70 \%$ de los casos. La edad promedio fue de 43,15 años (Gráfico 2).

En cuanto al tipo histológico el carcinoma de células escamosas fue el más frecuente, presentándose en 


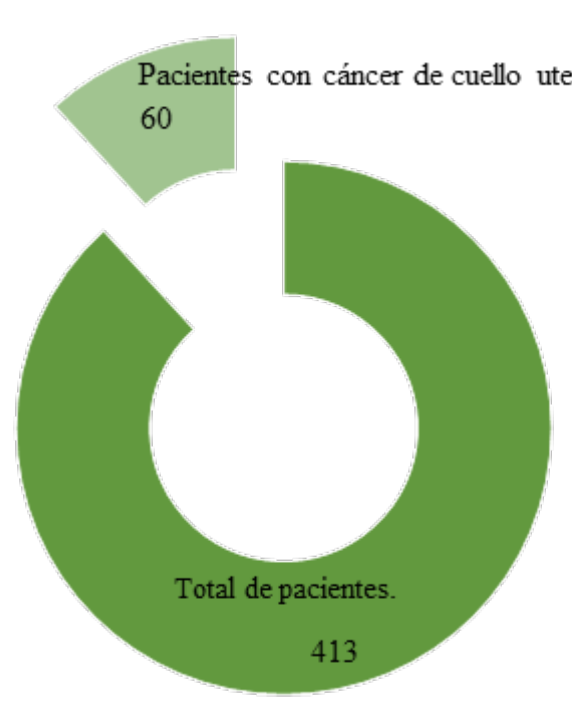

Incidencia de cáncer de cuello uterino: $14,52 \%$

Gráfico 1. Incidencia de cáncer de cuello uterino

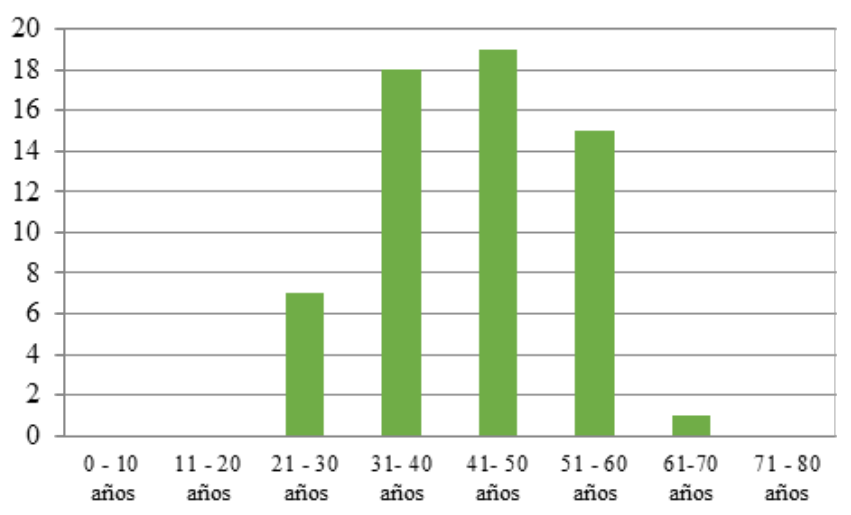

Gráfico 2. Distribución según grupos etarios

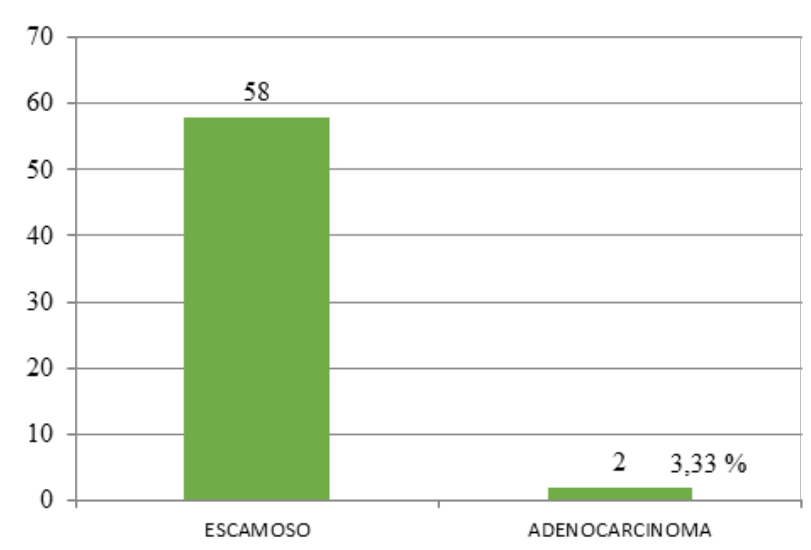

Gráfico 3. Distribución según el tipo histológico.
58 de las pacientes, lo que representó el 96,67 \% de los casos. El otro tipo histológico encontrado fue adenocarcinoma en 2 de las pacientes, que representó el 3,33\% de los casos (Gráfico 3).

La distribución por estadios se presenta en el gráfico 4. Destaca que se diagnosticaron 14 casos $(23,33 \%)$ en estadio IIB y 28 casos $(46,67 \%)$ en estadio IIIB.

Sobre el tratamiento, en 56 de los casos $(93,33 \%)$ se indicó radioterapia y quimioterapia y solo 4 pacientes $(6,66 \%)$ fueron sometidas a tratamiento quirúrgico (Gráfico 5).

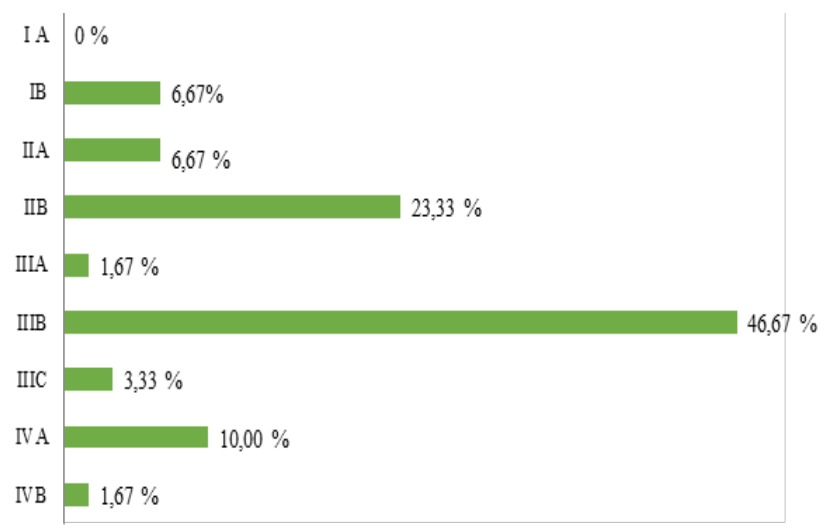

Gráfico 4. Distribución porcentual de pacientes según estadio del cáncer de cuello uterino al momento del diagnóstico

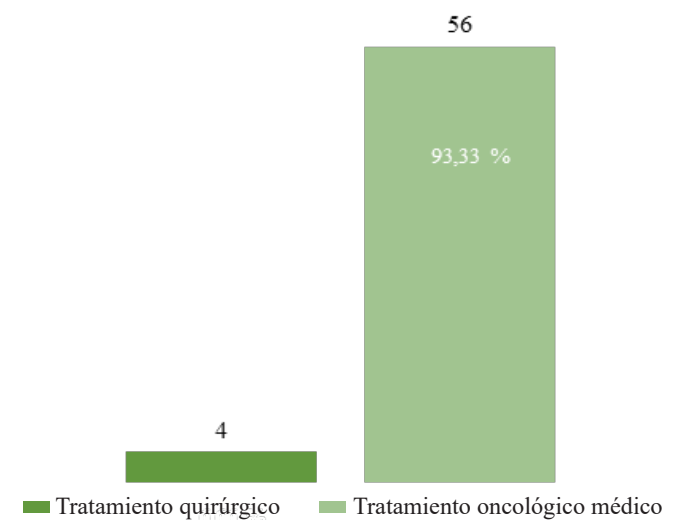

Gráfico 5. Distribución porcentual de pacientes según el tratamiento indicado. 


\section{CÁNCER DE CUELLO UTERINO. INCIDENCIA REGISTRADA EN EL HOSPITAL \\ “DR. DOMINGO LUCIANI” EN EL AÑO 2020}

\section{DISCUSIÓN}

Con un total de 604127 nuevos casos y 341831 muertes en 2020 a nivel mundial, el cáncer de cuello uterino se posiciona como el cuarto cáncer más frecuentemente diagnosticado y la cuarta causa de muerte en la población femenina (1). En países de bajos ingresos ocupa el segundo lugar en incidencia y mortalidad después del cáncer de mama; sin embargo, es el cáncer más comúnmente diagnosticado en 28 países y la principal causa de muerte por cáncer en 42 países, la gran mayoría de los cuales se encuentran en África subsahariana y el sudeste asiático (3). En Venezuela, de acuerdo a las estimaciones realizadas por IARC para el año 2020, el cáncer de cuello uterino ocupó el segundo lugar en incidencia por cáncer en la población femenina, con 3709 nuevos casos (4).

En el presente estudio se encontró que, en el Hospital "Dr. Domingo Luciani”, durante el año 2020, se diagnosticaron 60 nuevos casos, encontrando que $14,52 \%$ de las mujeres atendidas en la consulta de ginecología oncológica presentaron esta patología. Por otra parte, en Venezuela, en cuanto a las entidades geográficas, para 2012, Capote Negrín (9) encontró, en Distrito Capital y Miranda, 305 y 393 casos de cuello uterino, respectivamente.

De acuerdo al tipo histológico, el carcinoma de células escamosas representa aproximadamente el $80 \%$ de todos los casos de cáncer de cuello uterino y el adenocarcinoma el $20 \%$ (10). En el presente trabajo de investigación se determinó que el carcinoma de células escamosas fue diagnosticado en el 96,67\% de los casos y en 3,33 \% adenocarcinoma. Similarmente, Lobo y cols. (11) encontraron que el carcinoma de células escamosas es el tipo histológico más frecuente representando el $84 \%$ seguido por el adenocarcinoma con $10 \%$.
El grupo etario más afectado en este estudio, fue 41 a 50 años, con una edad promedio al momento del diagnóstico de 43,15 años, en contraposición a los datos reportados por el Instituto Nacional de Cáncer de los EE. UU., que describe como el grupo más afectado aquel con edades comprendidas entre 35 y 44 años, con $22,8 \%$ y una edad promedio al momento de diagnóstico de 50 años (12). Igualmente Capote Negrin (13) encontró en Venezuela, para el año 2012, que el grupo más afectado fue aquel entre 45 y 54 años de edad y Lobo y cols. (11) reportan una edad promedio de 53 años al momento del diagnóstico.

En cuanto al estadio FIGO de la enfermedad, en este estudio la mayoría de los casos fueron diagnosticados en estadios avanzados, principalmente estadios IIB y IIIB, con $23,33 \%$ y $46,67 \%$, respectivamente, y $90 \%$ de las pacientes diagnosticadas en estadios avanzados. Esto contrasta con las estadísticas publicadas por el Instituto Nacional de Cáncer de los EE. UU., quienes reflejan que el $44 \%$ de los casos se presentan con enfermedad confinada al útero (12).

En vista que en este estudio el mayor porcentaje de pacientes se diagnosticó en estadios avanzados, el tratamiento indicado fue la combinación de quimioterapia y radioterapia en el 93,33\% de los casos y solo en $6,67 \%$ de los casos fueron candidatas a tratamiento quirúrgico.

\section{CONCLUSIONES}

Se evidencia en el presente estudio que el cáncer de cuello uterino representa un porcentaje importante de consultas ginecológicas, que lamentablemente el diagnóstico se hace en estadios avanzados, afectando negativamente el pronóstico de estas pacientes y que se diagnostica con frecuencia en plena vida productiva de las mujeres afectadas, constituyendo un verdadero problema de salud pública y un caos para las familias y la sociedad. 


\section{REFERENCIAS}

1. International Agency for Research on Cancer. Global Observatory of Cancer-GLOBOCAN. Cancer Today [Internet]. Lyon: Cervix uteri; 2020 [consulta: 05/09/21]. Disponible en: https://gco.iarc.fr/today/data/ factsheets/cancers/23-Cervix-uteri-fact-sheet.pdf

2. Bhatla N, Berek JS, Cuello Fredes M, Denny LA, Grenman S, Karunaratne K, et al. Revised FIGO staging for carcinoma of the cervix uteri. Int J Gynaecol Obstet. 2019; 145(1):129-135. Doi: 10.1002/ijgo.12749.

3. Bray F, Ferlay J, Soerjomataram I, Siegel RL, Torre LA, Jemal A. Global cancer statistics 2018: GLOBOCAN estimates of incidence and mortality worldwide for 36 cancers in 185 countries. CA Cancer J Clin. 2018; 68(6):394-424. Doi: 10.3322/caac.21492.

4. International Agency for Research on Cancer. Global Observatory of Cancer-GLOBOCAN. Cancer Today [Internet]. Lyon: Venezuelan Bolivarian Republic of; 2020 [consulta: 05/09/21]. Disponible en: https:// gco.iarc.fr/today/data/factsheets/populations/862venezuela-bolivarian-republic-of-fact-sheets.pdf

5. Crosbie EJ, Einstein MH, Franceschi S, Kitchener HC. Human papillomavirus and cervical cancer. Lancet. 2013; 382(9895):889-99. Doi: 10.1016/S01406736(13)60022-7.

6. García J, Quinde V, Bucaram R, Sánchez G. Situación epidemiológica del cáncer cérvicouterino en el Ecuador. 2020. Rev Venez Oncol [Internet]. 2021 [consultado 05/09/21]; 33(2): 69-78. Disponible en: http://www. oncologia.org.ve/site/upload/revista/pdf/03._garcia_j_ (69-78).pdf

7. Small W Jr, Bacon MA, Bajaj A, Chuang LT, Fisher BJ, Harkenrider MM, et al. Cervical cancer: A global health crisis. Cancer. 2017; 123(13):2404-2412. Doi: 10.1002/cncr.30667.

8. Ries LAG, Melbert D, Krapcho M, Mariotto A, Miller BA, Feuer EJ, et al. SEER Cancer Statistics Review, 1975-2004 [Internet]. Instituto Nacional del
Cáncer. Bethesda, MD, 2007 [consultado 20/11/2020]. Disponible en: https://seer.cancer.gov/archive/ csr/1975_2004/

9. Capote Negrín L. Resumen del cáncer en Venezuela. Rev Venez Oncol [Internet]. 2015 [consultado 05/09/2021]; 27(4):256-268. Disponible en: http://www.oncologia. org.ve/site/upload/revista/pdf/10._capote_1_(256-268). pdf

10. National Comprehensive Cancer Network. NCCN Clinical Practice Guidelines in Oncology (NCCN Guidelines) [Internet]. Cervical Cancer (Version 1.2021); 2021 [consultado: 05/09/21]. https://www. nccn.org/professionals/physician_gls/pdf/cervical.pdf

11. Lobo J, Calderaro F, Jurado J, Escalona S, López A, Díaz Diego. Neoplasia maligna ginecológica. Incidencia en el nuevo milenio. Experiencia del Servicio oncológico hospitalario IVSS. Rev Venez Oncol [Internet]. 2019 [consultado 05/09/21]; 31(1):16-23. Disponible en: http://www.oncologia.org.ve/site/upload/revista/ pdf/03._lobo_j_(16-23).pdf

12. National Cancer Institute [Internet]. Cancer Stat Facts: Cervical Cancer. 2020 [consultado: 09/05/21]. Disponible en: https://seer.cancer.gov/statfacts/html/ cervix.html

13. Capote Negrín L. Resumen de las estadísticas de cáncer en Venezuela en el año 2012 [Internet]. Caracas: Sociedad Venezolana de Salud Pública. Red Defendamos la Epidemiología Nacional. 2015 [consultado: 12/05/21]. Disponible en: http://www.sogvzla. org.ve/sogvzla20186/saciverfotonoticiaconvista. php?id $=0000000142$ 